\title{
The Use of Time Lapse Imaging to Study the Effect of Hyperphosphorylated Tau
}

\author{
Christopher P. Corbo, M. Eugenia Alaniz, Mina Farid, Alejandra del C. Alonso
}

CUNY, Staten Island

A collective group of neurodegenerative diseases, including Alzheimer's disease, are commonly referred to as tauopathies because of the pathological role played by the neuronal microtubule associated protein, tau. These diseases are histopathologically characterized by neurofibrillary tangles (NFTs) composed of hyperphosphorylated forms of tau. Normally this phosphoprotein plays a key role in the stabilization of the microtubule networks. When hyperphosphorylated tau no longer binds to microtubules, it begins to aggregate. Here our objective is to study the influence of tau phosphorylation at Thr 212, Thr 231, and Ser 262 (Ptau) on tau self-assembly and the effect of its expression on the cells. Pseudophosphorylated tau was generated by site directed mutagenesis, switching the above mentioned amino acid residues with glutamic acid, to mimic the effect the phosphorylation would have on the protein. We found that pseudophosphorylated tau aggregates in cells when Thr 212 is mutated to Glu, suggesting that phosphorylation at this site facilitates tau self-assembly. The expression of tau pseudophosphorylated at Thr212, Thr231, and Ser262 triggers caspase 3 activation in as many as $85 \%$ of the transfected cells and apoptosis to a lesser degree. These findings suggest that tau phosphorylation at Thr 212 facilitates tau self-aggregation, and that the combination of phosphorylation at Thr212, 231 and Ser262 in the same tau molecule can trigger a toxic reaction. In order to study the effects of hyperphosphorylated tau protein on microtubules dynamics, live cell imaging is of absolute necessity.

This project set out to characterize the impact that hyperphoaphorylated tau has on the normal cellular processes in the cell including microtubule dynamics, membrane integrity and the proteins ability to enter the nucleus through the use of time-lapse imaging techniques.

To accomplish this objective, Chinese hamster ovarian fibroblast (CHO) cells were transfected with constructs expressing pseudophosphorylated tau, mimicking phosphorylation at Thr 212, Thr 231, and Ser 262, was inserted in reading frame with GFP in an expression vector. Transfected cells were imaged under the confocal laser scanning microscope using time-lapse imaging at intervals between three and four seconds. Membrane studies were conducted using vital fluorescent dyes specific to the cell membrane (Invitrogen Inc.). Time-lapse movies allowed for the analysis of tau's interaction with microtubules as well as its ability to aggregate within the cells. Further analysis will be conducted using taxol and colcemid to analyze the function of discern if these apoptotic events are due to microtubule breakdown or the P-tau itself.

Important events seen during the imaging all provide strong evidence of cellular toxicity. This evidence includes a malformed microtubule structure and the movement of P-tau into the nucleus. The cytoplasm of the transfected cells were filled with intracellular tau aggregates as well as the accumulation of intracellular vacuoles. Many of these vacuoles, especially those smaller in size are actively moving around in the cytoplasm. This movement is most likely diffusion as opposed to movement due to a lack of microtubules. After 36 hours post transfection, the plasma membrane shows advanced signs of apoptosis in that the membrane begins to bleb out. The rate of blebbing is easily seen through the time-lapse imaging. This blebbing of the membrane leads to a decrease in the size of the cells. Furthermore, we studied the effects of P-tau on microtubule dynamics as seen when comparing the Wt tau with the P-tau cells. 

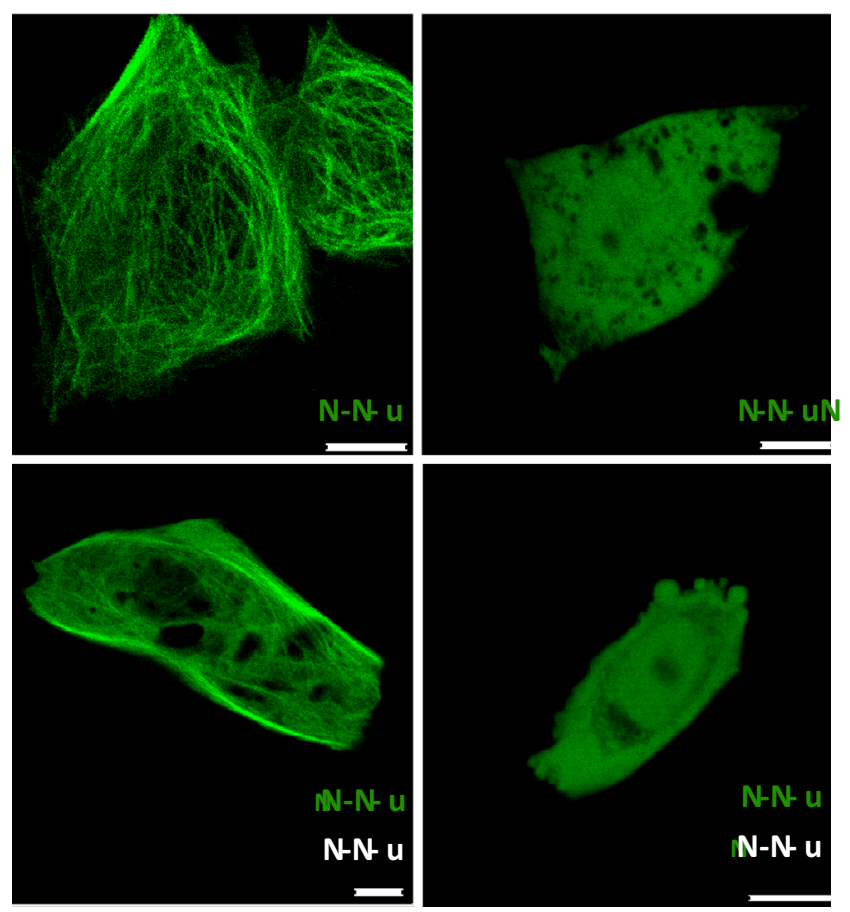

Figure 1 - $\mathrm{CHO}$ cells were transfected with: a) normal wild type tau (N-tau), b) P-tau or c) the mixture of fluorescent $\mathrm{N}$-tau with non-fluorescent $\mathrm{P}$-tau or d) non-fluorescent $\mathrm{N}$-tau with fluorescent $\mathrm{P}$-tau. Healthy microtubule structures are easily recognized when cells were transfected with $\mathrm{N}$-tau. Cells transfected with P-tau. showed unusual, large vacuoles as well as a lack of microtubule structure and the appearance of fluorescence in the nucleus. Cells transfected with both normal tau and P-tau had an unhealthy appearance. Cells transfected with a mixture of fluorescent $\mathrm{N}$-tau with non-fluorescent $\mathrm{P}$-tau showed healthy microtubules in the periphery of the cell, but the homogeneous fluorescence and vacuoles in the cytoplasm. In cells transfected with non-fluorescent wild type with fluorescent $\mathrm{P}$-tau no healthy microtubules are recognizable and apoptotic membrane blebbing can be seen. All scale bars represent 8 um.

The time-lapse images provide a dynamic view of the effects caused by the hyperphosphorylated tau including the membrane blebbing, tau movement into the nucleus, intracellular aggregates and vacuoles. This method of imaging provides new insights into the disease mechanisms not seen in fixed samples. 